\title{
Communication, Renegotiation, and the Scope for Collusion
}

\author{
By David J. CoOper AND KaI-Uwe KüHN*
}

\begin{abstract}
We study the effect of communication in an experimental game where cooperation is consistent with equilibrium play if players share an understanding that cheating will be punished. Consistent with communication acting as a coordinating device, credible preplay threats to punish cheating are the most effective message to facilitate collusion. Promises to collude also improve cooperation. Credible threats do not occur in a treatment with a limited message space that permits threats of punishment. Contrary to some theoretical predictions, renegotiation possibilities facilitate collusion. (JEL C71, C73, D83, L12)
\end{abstract}

The Finns will respect the Spanish dominance in Spain if ENCE really increase their prices in other countries: If Finncell learn about prices below US $\$ 360$ also in the future, they will reconsider their policy as to sales in Spain!

— European Commission, 1985

The preceding quote is from a document found in the European Woodpulp case
(Decision $85 / 202 /$ EEC, 1985, published in OJ L85/1). Finncell, the joint sales
organization for Finnish producers, promises to abide by a collusive agreement if
ENCE, the leading Spanish producer of wood pulp, does so as well, but threatens
to punish ENCE in the future if it departs from the agreement. Communications
containing explicit threats and promises of this sort are understood to be at the heart
of illegal cartel activities. The per se prohibitions on price fixing under the Sherman
Act in the United States and Article 101 of the Treaty of Rome in the European
Union are effectively prohibitions on such conversations, and enforcement focuses
on discovering evidence of communication and explicit agreements. Despite this
emphasis by antitrust authorities, incriminating communication commonly occurs
within cartels, as is well-documented in case and field evidence (e.g., Genesove and

\footnotetext{
* Cooper: Department of Economics, Florida State University, 265 Bellamy Building, Tallahassee, FL 32306 (e-mail: djcooper@fsu.edu) and School of Economics, University of East Anglia (e-mail: D.J.Cooper@uea.ac.uk); Kühn: Department of Economics, University of Michigan at Ann Arbor, 238 Lorch Hall, 611 Tappan St, Ann Arbor, MI 48109 (e-mail: kukuhn@umich.edu). We would like to thank the National Science Foundation (SES0720993) for funding these experiments. Angelo Benedetti, E. Glenn Dutcher, John Jensenius, Cortney Rodet, Micah Sanders, Elena Spatoulos, and Evan Starr provided valuable research assistance on this project. We would like to thank two anonymous referees, Tim Cason, Gary Charness, Martin Dufwenberg, Guillaume Fréchette, Larry Samuelson, Steve Salant, Yossi Spiegel, Roberto Weber, Mike Whinston, and various seminar participants for their helpful feedback. The authors are solely responsible for any errors in this manuscript.

${ }^{\dagger}$ Go to http://dx.doi.org/10.1257/mic.6.2.247 to visit the article page for additional materials and author disclosure statement(s) or to comment in the online discussion forum.
} 
Mullin 2001). The frequency of interfirm communication about collusion in the face of large fines suggests that it must be a valuable tool for establishing collusive outcomes. However, it is not well understood why this is the case and what needs to be said to make collusion successful.

Price collusion between firms is just one example where repeated interaction makes cooperation consistent with equilibrium play, even though agents have incentives to behave noncooperatively in the short run. A common element across such cases is that cooperation can be enforced in equilibrium by the threat to switch from cooperative behavior to noncooperative behavior after a deviation is observed. We frame our discussion in terms of price collusion, but our insights about the role of communication in improving cooperation are applicable to other cases where cooperative equilibria have this structure. This includes examples where cooperation is socially desirable, such as implicit contracting, team production, and the provision of local public goods.

This intuition has been formalized in the theory of infinitely repeated games (Abreu 1988; Abreu, Pearce, and Stacchetti 1990). The theory does not model communication, but implies that collusion relies on firms solving a coordination problem: cooperation can only be supported if players know that deviations will be punished by switching from a high-payoff to a low-payoff continuation equilibrium. The literature typically assumes that coordination on the necessary contingent strategies is easily achieved. However, experimental evidence from related coordination games shows that subjects often fail to reach a Pareto optimal equilibrium in the absence of an explicit coordination device (Van Huyck, Battalio, and Beil 1990). Communication dramatically increases the likelihood of coordination on an efficient equilibrium (Cooper et al. 1992; Blume and Ortmann 2007; Brandts and Cooper 2007). Coordination on a collusive equilibrium is far more complex, requiring agreements not just on a single action but on entire contingent plans. It is not obvious that communication can be as effective a coordination device in such settings.

At the same time experimental evidence suggests that communication may have a role beyond coordination, since it can lead to more cooperative outcomes in oneshot (or finitely repeated) games where cooperation is not an equilibrium outcome (e.g., Dawes, MacTavish, and Shaklee 1977; Isaac and Walker 1988; Cason and Mui 2009; Charness and Dufwenberg 2006). There are many explanations why communication makes subjects more willing to cooperate in the absence of monetary incentives, including increased group identity, aversion to lying (or guilt aversion, which is slightly different), ${ }_{1}^{1}$ and improved understanding of the mutual benefits of cooperation. If communication primarily improves collusion through such channels, the mechanism of rewards and punishment in the continuation game may be unnecessary to sustain cooperative outcomes. Such a finding would reduce the practical relevance of the theory of repeated games which explains cooperation as an equilibrium phenomenon that relies on this mechanism.

\footnotetext{
${ }^{1}$ These concepts are closely related to results from the psychology literature finding that communication allows players to make promises which are binding due to strong norms, both internal and social, against violating commitments (Kerr and Kaufman-Gilliland 1994).
} 
We explore these different explanations for how communication might foster collusion through a series of experiments that vary the type of communication available. Subjects play a sequence of two-period collusion games with random rematching between games. Collusion (mutual choice of a high price) can be supported in the first period as part of a subgame perfect equilibrium, but only if deviations are punished by play of a Pareto inferior equilibrium in the second period. The two-period collusion game therefore captures the main strategic features of infinitely repeated collusion games while avoiding a number of methodological problems occurring for experiments on communication in indefinitely repeated games.

In an initial phase without communication, first-period play quickly collapses to the noncooperative equilibrium. ${ }^{2}$ Communication is then added for the second phase of the experiment. If communication is limited to pregame statements of intent to collude in Period 1 with no possibility of specifying a punishment scheme for deviations, an initial increase in collusion is followed by a collapse back to the noncooperative equilibrium. This is in line with results reported by Holt and Davis (1990). Adding the possibility of specifying a punishment scheme without otherwise expanding the message space does not improve matters, as play still collapses back to the noncooperative equilibrium. When a rich pregame message space is used - subjects have access to a chat window and can send and receive unlimited messages - there is again an initial burst of collusive behavior followed by gradual deterioration. Unlike the treatments with limited message spaces, this decline slows and is eventually reversed in the pregame chat treatment. By the end of the experiment, collusive behavior returns to its initial high levels. When renegotiation is allowed by adding chat between periods of the game, collusion is even more common and never exhibits a decline. This contradicts the unambiguous theoretical prediction for the game we implement: renegotiation should eliminate all collusion by making it impossible to credibly commit to punish cheating.

Detailed analysis of the chat content identifies two channels by which communication leads to persistent collusion in the treatment with preplay chat: credible threats and promises. Subjects who either send or receive credible threats (i.e., a threat of punishment that, if believed, makes it incentive compatible to abide by a collusive agreement) that noncollusive play will be punished are significantly less likely to cheat on collusive agreements. The effect is large. Sending a credible threat is estimated to lower the probability of cheating by 40 percent and receiving a credible threat lowers the probability of cheating by 26 percent. Credible threats are by far the most effective type of communication for bolstering collusion in the treatment with preplay communication. Underlying the effectiveness of credible threats are changes in the incentive to collude: Generally we observe higher payoffs for cheating on a collusive agreement than complying with it, but this reverses when a credible threat is sent or received.

The powerful effect of credible threats supports the role of communication as a coordination device to achieve a collusive equilibrium consistent with the standard

\footnotetext{
${ }^{2}$ The payoffs in our game are designed to make it likely that collusion will fail in the absence of communication, but this is not a universal feature of collusion games. Dal Bó and Fréchette (2011) study which features of the payoff table make collusion likely.
} 
theory. If collusion was observed but such punishment was rarely mentioned or if it played a smaller role in fostering collusion than other types of communication, this would have been a cause for skepticism about the theory itself. Our result complements earlier experimental work on collusion by Dal Bó (2005) which demonstrated that collusive play is more likely when players operate "under the shadow of the future." We show that the "shadow of the future" becomes much more important when explicit threats of punishment can be made.

Given the importance of credible threats in the preplay chat treatment, it is surprising that collusion is so low in the treatment with a limited message space that allows for threats of punishment. The messages sent in this treatment indicate that the problem is not a failure to threaten punishment, but instead a failure to specify sufficiently harsh punishments for cheating to be unprofitable. Limited message treatments are therefore not a good substitute for more natural conversations that take place with open chat. Limited message treatments may miss the types of messages that actually matter and the available messages are used differently than they would be in a natural conversation.

The second channel through which communication boosts collusion in the treatment with preplay chat is promises of trustworthy behavior. Sending promises of trustworthy behavior is associated with a significant decrease in cheating by the sender on collusive agreements. The marginal effect is less than half of the effect of sending a credible threat, reducing cheating by 19 percent. On the surface this lines up with the results of Charness and Dufwenberg (2006), as well as the psychology literature (see Kerr and Kaufman-Gilliland 1994, for a summary) finding that promises increase cooperation. However, in our setting neither an aversion to lying nor guilt aversion is necessary to explain the self-commitment effect of promises: subjects who send explicit promises face sufficiently strong punishment for cheating that collusion becomes incentive compatible. Surprisingly, this self-commitment is not valuable to the subject sending the promise since it does not reduce cheating by the individual receiving the promise.

In the treatment allowing for renegotiation, the second-period chat provides a clear explanation why collusion is more successful than in any other treatment. Consistent with renegotiation theory, players try to avoid administering a punishment following cheating and these attempts have some success. As a result, average monetary punishments after deviations from collusive agreements are the weakest of all communication treatments. However, this is counteracted by a second important effect of allowing chat between the two periods of the game: individuals who are cheated can reproach those who cheated them. They seize upon this opportunity with high frequency and great enthusiasm. The availability of an inexpensive and effective form of punishment in the treatment with renegotiation provides a good explanation for the high and stable levels of collusion achieved in this treatment. ${ }^{3}$

\footnotetext{
${ }^{3}$ This is similar to the effect of nonpecuniary punishments (disapproval points) in public goods games (Masclet et al. 2003). The effect here is more persistent, possibly due to the richer set of verbal punishments available. Likewise, Xiao and Houser (2005) find that the possibility of verbal punishment reduces rejection rates in ultimatum games.
} 
The paper is organized as follows. In Section I we discuss collusion theory and the theory of communication in games. Section II describes the experimental design in detail. In Section III we present the experimental results. We first analyze the benchmark behavior when there is no communication possible and then look at the short and long run treatment effects of various communication treatments. We then go into more detail on the content analysis of the preplay chat treatment and the renegotiation treatment. Section IV concludes the paper and discusses the relationship between our work and field studies of communication and collusion.

\section{Communication and the Theory of Collusion}

The standard theoretical approach to price collusion is quite simple conceptually: collusion can be supported at a price greater than the competitive price if the short-run gain from undercutting is less than the long run losses induced by future punishment involving a switch from collusive to competitive behavior. What is critical for the argument is that both the promise of future collusion as a reward for past collusive behavior and the threat of future competitive behavior as a punishment for a past deviation are credible in the sense that they involve equilibrium play. A credible threat therefore requires a coordinated switch between different equilibria of the continuation game. A central question in our study is how communication helps players coordinate on the contingent play necessary for a collusive equilibrium. This section develops predictions on what kind of communication we should observe if cheap talk can be used as a coordination device in collusion games.

To fix ideas we discuss the specific two-period game employed in our experiments. The following two matrices show the row player's payoffs for Period 1 and Period 2, respectively. The payoff structure is symmetric, and the row player's payoff for actions $(i, j)$ equal the column player's payoffs for action $(j, i)$.

Period 1

\begin{tabular}{lrcc}
\hline & Low & Medium & High \\
Low & 15 & 54 & 54 \\
Medium & -24 & 45 & 114 \\
High & -24 & -24 & 60
\end{tabular}

Period 2

\begin{tabular}{lccr}
\hline & Low & Medium & High \\
Low & 30 & 56 & 56 \\
Medium & 4 & 90 & 96 \\
High & 4 & 4 & 120 \\
\hline
\end{tabular}

The Period 1 stage game can be interpreted as a standard Bertrand game in which firms have a choice between three possible prices and have a sunk cost of 24 . The unique Nash equilibrium of the Period 1 stage game played in isolation is $(\mathrm{L}, \mathrm{L})$. The Period 2 stage game is derived from the continuation profits of an infinitely repeated version of the Period 1 stage game with discounting, as described in online Appendix $\mathrm{C}$. This results in a coordination game in which there are three pure strategy equilibria, $(\mathrm{L}, \mathrm{L}),(\mathrm{M}, \mathrm{M})$, and $(\mathrm{H}, \mathrm{H})$. These equilibria are Pareto ranked with $(\mathrm{H}, \mathrm{H})$ being the Pareto dominant equilibrium.

We refer to the full two-period game as the Two-Period Bertrand Game (TPBG). The necessary incentive conditions are satisfied so that $(\mathrm{L}, \mathrm{L}),(\mathrm{M}, \mathrm{M})$, or $(\mathrm{H}, \mathrm{H})$ 
can all be sustained in the first period of a subgame perfect equilibrium if players play (L, L) in Period 2 after any deviation in Period 1 and $(\mathrm{H}, \mathrm{H})$ otherwise. Asymmetric Period 1 outcomes $(H, L)$ and $(M, L)$ (and their permutations) can also be sustained in this way but not $(H, M) .{ }^{4}$ All collusive equilibria of the TPBG, defined as equilibria that yield an outcome other than $(\mathrm{L}, \mathrm{L})$ for Period 1 , require that players use the first-period outcome as a coordination device for play in the second period. This captures the essential structure of all theories of collusion based on infinitely repeated games (Abreu 1988; Abreu, Pearce, and Stacchetti 1990) or finitely repeated games (Benoit and Krishna 1985).

For preplay communication to have a systematic impact on outcomes, players must take messages to have meaning. But players cannot believe just any message because opponents may have an incentive to lie in order to induce favorable behavior. We assume that two features of communication are necessary for credible communication. First, a message is credible if each player has an incentive to do what he proposes as long as he expects the proposal to be believed by the other player. ${ }^{5}$ Second, a pair of proposals from two different players should be considered credible only if the proposals are compatible. These two conditions are equivalent to requiring agreement on a Nash equilibrium of the game. Since all Nash equilibria in the TPBG that support Period 1 prices above $L$ involve contingent play in Period 2 (i.e., play in Period 2 varies depending on the Period 1 outcome), these assumptions about credibility predict that communication can facilitate collusion only if messages specifying contingent strategies are available and used. Going further, only messages that threaten to punish cheating with play of Low in Period 2 provide credible support for a proposal to play $(\mathrm{H}, \mathrm{H})$ in Period 1.

If communication is used as a coordination device prior to Period 1, we should also expect subjects to go further and coordinate on an equilibrium whenever they can communicate. This raises the issue of renegotiation prior to Period 2. Renegotiation theory is built on the assumption that players will use an unspecified coordination device to achieve a Pareto undominated continuation equilibrium after any history of play. ${ }^{6}$ Suppose players in the TPBG can communicate and hence coordinate before both periods. Since continuation equilibria in the TPBG are Pareto ranked, the intuition underlying renegotiation theory implies players will agree on and play $(\mathrm{H}, \mathrm{H})$ in Period 2 regardless of the Period 1 outcome. Messages prior to Period 1 that specify a different equilibrium for Period 2 should therefore be ignored because rational individuals will anticipate the impact of renegotiation prior to Period 2. It

\footnotetext{
${ }^{4}$ Note that $(\mathrm{H}, \mathrm{H})$ can only be sustained in Period 1 if $(\mathrm{L}, \mathrm{L})$ is played in Period 2 after a deviation and $(\mathrm{H}, \mathrm{H})$ otherwise. All other Period 1 subgame perfect equilibria can also be sustained by playing $(\mathrm{L}, \mathrm{L})$ after a deviation and $(\mathrm{M}, \mathrm{M})$ otherwise in Period 2. The only Period 1 outcome that can be sustained by playing $(\mathrm{M}, \mathrm{M})$ after a deviation and $(\mathrm{H}, \mathrm{H})$ otherwise is $(\mathrm{M}, \mathrm{M})$ in Period 1.

${ }^{5}$ This condition corresponds to the concept of "self-commitment" in Aumann (1990). Aumann also requires that messages are "self-signaling": the sender only wants the message to be believed if he is telling the truth. This condition is much more controversial. As Farrell and Rabin (1996) point out, this condition leads to unlikely predictions about the effectiveness of communication in stag-hunt games and cannot be satisfied in collusion games.

${ }^{6}$ See Bernheim, Peleg, and Whinston (1987); Bernheim and Ray (1989); and Benoît and Krishna (1993) for finitely repeated games and van Damme (1989); Farrell and Maskin (1989); and Abreu, Pearce, and Stacchetti (1993) for supergames. The assumption that a Pareto undominated equilibrium will be chosen is not generally accepted in the cheap talk literature for cases, unlike the TPBG, where equilibria cannot be Pareto ranked since there is a conflict of interest in these cases (Farrell and Rabin 1996).
} 
follows that messages prior to Period 1 cannot credibly specify a contingent strategy for Period 2. No collusion should therefore occur in Period 1 of the TPBG if we allow for communication as a coordination device between Period 1 and Period 2 play.

It is not a universal property of collusion games that allowing renegotiation eliminates the possibility to collude. This occurs in the TPBG because the equilibria of the Period 2 game can be Pareto ranked. The scope for collusion under renegotiation is significantly wider when there are multiple asymmetric continuation equilibria that cannot be Pareto ranked. The TPBG is designed to make the predicted effect of renegotiation as stark as possible, yielding a clean test of the main idea underlying all models of renegotiation: the ability to renegotiate will eliminate the use of Pareto dominated continuation equilibria and therefore limit the outcomes that can be supported as equilibria in the full game.

Several other features of the TPBG are designed to make the results easier to interpret. The equilibrium (M, M) is risk dominant (Harsanyi and Selten 1988) in the second-period game. This makes it relatively likely that $M$ will be chosen in the absence of an explicit coordination device. If, in contrast, $(\mathrm{H}, \mathrm{H})$ were both Pareto and risk dominant, $H$ would be a natural default choice in Period 2. Players could then easily coordinate on $(\mathrm{H}, \mathrm{H})$ without an explicit coordination device and it would be more difficult to identify the effect of communication as a coordination device when renegotiation is allowed. Making ( $\mathrm{M}, \mathrm{M})$ risk dominant also helps to identify contingent behavior. Since collusion at $(\mathrm{H}, \mathrm{H})$ in Period 1 can only be supported by a switch from $(\mathrm{H}, \mathrm{H})$ to $(\mathrm{L}, \mathrm{L})$ in Period 2, more effective communication should move Period 2 play away from $(\mathrm{M}, \mathrm{M})$ to the extremes. Note that $(\mathrm{L}, \mathrm{L})$ in Period 2 is unlikely to occur due to coordination failure rather than a conscious decision to punish cheating because it is both Pareto and Risk dominated.

More generally, the TPBG provides strong incentives in the sense that collusion at $H$ is highly beneficial, strong punishments are needed to maintain collusion in equilibrium, and the loss from cheating and being punished in equilibrium is large. The payoff from play of $(\mathrm{H}, \mathrm{H})$ is 33 percent larger in each period than the payoff from play of $(\mathrm{M}, \mathrm{M})$, collusion at $H$ in Period 1 can only be supported as an equilibrium outcome via reversion to $(\mathrm{L}, \mathrm{L})$ in Period 2 which reduces payoffs by 75 percent compared to play of $(\mathrm{H}, \mathrm{H})$, and the payoff from colluding at $(\mathrm{H}, \mathrm{H})$ in both periods is 25 percent greater than the payoff from defection to $M$ in Period 1 followed by reversion to (L, L) in Period 2 (180 versus 144). Subjects are given strong incentives to reach and abide by collusive agreements, but the punishment called for by the equilibrium is sufficiently harsh for both players that it is unlikely to be undertaken lightly.

\section{Experimental Design}

\section{A. General Design}

Subjects play 20 rounds of the TPBG in all treatments. A "round" refers to an entire play of the TPBG while a "period" refers to one of the two games played within a single round of the TPBG. Subjects are randomly matched with a new 
opponent in each round. Sessions are sufficiently large (minimum of 20 subjects) that it is unlikely that there are repeated game effects between rounds.

For the first ten rounds in all treatments subjects play the TPBG without any communication. This is sufficient for subjects to understand the experimental interface, payoff tables, and main strategic issues in the TPBG before attempting to master use of communication. Having ten rounds of play before introducing communication also allows play to converge to the one shot Nash equilibrium in the first period, making the task facing subjects in Rounds 11-20 more challenging. Treatments vary by the type of communication available in Rounds 11-20.

\section{B. Use of the TPBG}

By using the TPBG our design differs from earlier work on collusion that used indefinitely repeated games as proxies for supergames (e.g., Roth and Murnighan 1978; Dal Bó 2005; Dal Bó and Fréchette 2011; Duffy and Ochs 2009). The simplicity of a two-period game has significant methodological advantages for a study that focuses on communication and learning. The most important is speed of play and learning. Subjects go through a lengthy learning process, and our conclusions would differ substantially if this process lacked adequate time to converge. Generating the requisite experience is nontrivial since chat slows down play significantly and we limited ourselves to two hour sessions, including instructions, to avoid subject fatigue. The TPBG is sufficiently simple that most sessions were completed within two hours, even with chat, and play largely stabilized by the end of Round 20. Another advantage provided by the TPBG's simplicity is that we can make sharp theoretical predictions, as described in Section I, about what type of proposals for play of $(\mathrm{H}, \mathrm{H})$ in Period 1 are credible and what equilibria are renegotiation proof. Finally, using a relatively simple game helps with the statistical evaluation of the outcomes. Analyzing the content of messages is a daunting task even when the number of types of relevant messages that can be sent is small. If we expand the strategy space, and by extension increase the number of relevant message types, the analysis quickly becomes intractable.

This is not to say that use of the TPBG as a proxy for a supergame is riskless. Our main concern follows from the fact that a collusive equilibrium depends on players' behavior changing in Period 2 based on the outcome of Period 1. This requires subjects to see a connection between the two periods of the TPBG. Because the TPBG uses different games for the two periods, it is plausible that subjects will have more difficulty seeing the connection between periods than in an indefinitely repeated game where the payoff matrix is fixed. However, as documented below, we observe no shortage of contingent play in the four treatments with communication, and nothing in the data suggests that a lack of contingent play drives our qualitative results.]

\footnotetext{
${ }^{7}$ See Fréchette and Yuksel (2013) for a comparison of four different methods of implementing supergames in the laboratory, including our method and random termination. Levels of Period 1 cooperation are similar across all four methods, with our method finishing in the middle of the pack. Unlike our data, they observe relatively little contingent play in the final period (i.e., the coordination game). While there are a number of differences between the studies, we suspect that the differing results reflect differences in how the payoff tables were constructed for the
} 
In a follow-up study, we replicated results from the TPBG with a three period version of the game (stage game, stage game discounted, and coordination game). If contingent play is suppressed in the TPBG because the relationship between Periods 1 and 2 is not obvious to subjects, this should matter less in the three period game and cooperation should be higher. Instead, we find that the change to a longer game has no impact on Period 1 play. This gives us greater confidence that the results of our experiment are not sensitive to the number of periods in the game.

\section{The Communication Treatments}

We compare play in five communication treatments: No Communication, Period 1 Limited Communication, Period 1 Limited Communication with Contingencies, Preplay Chat, and Renegotiation. The following subsection describes each of these treatments. Recall that subjects play 20 rounds of the TPBG in all treatments. A "round" refers to an entire play of the two-period game while a "period" refers to one of the two stage games played within a single round of the TPBG. For the first ten rounds in all treatments subjects play the TPBG without any communication, so treatments only vary the type of communication that is possible in Rounds 11-20. All treatments have a pause following Round 10 while new instructions are read.

(i) No Communication (N Treatment).- The rules for Rounds 11-20 are identical to those in Rounds 1-10, with no communication between players. The $N$ treatment is included as a control to exclude the possibility that collusion in Round 11 is caused by a restart effect. In the pause following Round 10 it is announced that the games for Rounds 11-20 will use the same rules as for Rounds $1-10$.

(ii) Period 1 Limited Communication (P1 Treatment).- The P1 treatment gives subjects the opportunity to send a message prior to the beginning of Period 1. The message space is limited to suggesting actions for Period 1. Specifically, subjects are given the prompt, "I think we should choose the following in Period 1." They are asked to choose between "Low," "Medium," "High," or "No Response" for both "My Choice" and "Your Choice." Messages are chosen simultaneously and each player is shown both parts of both players' messages at the same time as choices are made for Period 1. The feedback at the end of Period 1 reiterates the messages as well as reporting the outcome for Period 1. Subjects cannot send any messages about their intent for Period 2.

(iii) Period 1 Limited Communication with Contingencies (P1C Treatment).In addition to specifying what actions should be chosen for Period 1, subjects in the $P I C$ treatment also indicate what actions should be chosen for Period 2 subject to the outcome for Period 1. The set of possible messages about Period 2 is limited: subjects are prompted "[i]f we choose the preceding

coordination game. Recall that the Period 2 table was constructed with (M, M) as the risk-dominant equilibrium, to make it more likely that contingent behavior could be detected. 
[i.e., the actions the subject has specified should be chosen for Period 1] in Period 1, I think we should choose the following in Period 2" and "[i]f we DO NOT choose the preceding [i.e., the actions the subject has specified should be chosen for Period 1] in Period 1, I think we should choose the following in Period 2." Limiting the message space simplifies the problem facing subjects while still making it possible to send a credible message that supports collusion in Period 1. Subjects are shown both players' messages, in full, at the same time as choices are made for Period 1. The messages are displayed again as part of the feedback for Period 1.

(iv) Preplay Chat (PChat Treatment).-At the heart of our experimental design are the two chat treatments. Starting in Round 11, the PChat treatment allows players to communicate using the chat option in version 3.1 of z-tree (Fischbacher 2007). This is very similar to using an IM program. Continuous back-and-forth communication is possible until one of the players makes a decision for the period. Subjects are given no guidance on how the chat should be used or what they might say, although it is fairly obvious that it is meant for discussing the game.

(v) Renegotiation (RChat Treatment).-Communication before Period 1 occurs in the RChat treatment in exactly the same way as in the PChat treatment. The RChat treatment differs from the PChat treatment by also allowing communication through a chat window after first-period actions are observed and before second-period actions are chosen, making renegotiation possible.

\section{Initial Hypotheses}

Our initial hypotheses for Period 1 play are derived from the discussion of communication and collusion in Section I. While it is possible to achieve tacit collusion in an experimental collusion game without communication, the TPBG is designed to make this unlikely in the $N$ treatment. ${ }^{8}$ The limited message space available in the $P 1$ treatment makes it impossible to reach a credible agreement to collude in Period 1. We therefore did not expect long term collusion in this treatment. Consistent with earlier results of Holt and Davis (1990), we expected an initial increase in collusion when communication was introduced since it takes some time to learn whether messages calling for Period 1 collusion are credible. The $P l C$ treatment allows for credible agreements to collude, so higher and more stable collusion was expected than in the $P 1$ treatment. For the same reason, the $P C h a t$ treatment was also expected to yield higher and more persistent collusion than the $P 1$ treatment. The rich communication available in the PChat treatment provides more scope for behavioral factors such as trust and guilt to have an effect, so we anticipated higher and more

\footnotetext{
${ }^{8}$ Collusion at $H$ is not risk dominant. Dal Bó and Fréchette (2011) find that making collusion at $H$ risk dominant is necessary but not sufficient to guarantee successful collusion, so we did not expect collusion at $H$ in the absence of communication.
} 
TABle 1 -Summary of Treatments

\begin{tabular}{lrrrrr}
\hline \hline & N & P1 & P1C & PChat & RChat \\
\hline Number of sessions & 3 & 3 & 3 & 3 & 3 \\
Number of subjects & 64 & 68 & 74 & 64 & 76 \\
First-period limited messages & & $\checkmark$ & $\checkmark$ & & \\
Contingent messages about Period 2 & & & $\checkmark$ & & $\checkmark$ \\
First-period chat & & & & $\checkmark$ \\
First- and second-period chat & & & &
\end{tabular}

stable collusion in the PChat treatment than in the $P 1 C$ treatment. $9^{9}$ The intuition underlying theories of renegotiation predicts that allowing chat between Periods 1 and 2 should undermine collusion in Period 1 and lead to coordination on $H$ in Period 2. We therefore expected to see less collusion in the RChat treatment than in the PChat treatment.

\section{E. Procedures}

The experiments were conducted at Case Western Reserve University using subjects recruited via e-mails sent to all undergraduates. Sessions were run in a computerized laboratory using z-Tree (Fischbacher 2007), and took between $1 \frac{1}{2}$ and 2 hours. Average earnings were slightly more than $\$ 20$, including a $\$ 6$ show-up fee. Subjects were paid their total earnings from all 20 rounds of the TPBG. Payoffs were denominated in experimental currency units (ECUs), converted to dollars at a rate of 130 ECUs equal $\$ 1$. Table 1 summarizes the number of subjects and sessions for each treatment. There are three sessions for each treatment with at least 20 subjects per session.

The instructions (see online Appendix E) were read to the subjects, and were also shown on the subjects' computer screens. Several times the payoff tables were projected on an overhead screen for examples, making the payoffs common knowledge. The matching for this experiment is relatively complex (fixed matching within a round, random rematching between rounds), so this point was emphasized. Following the instructions, subjects took a quiz testing their ability to read the payoff tables and their understanding of the instructions.

The experimental materials are framed using abstract language. For example, the materials do not refer to prices. Subjects choose between "A," "B," and "C" in Period 1 and between "D," "E," and "F" in Period 2, with the three labels in each period corresponding to low, medium, and high prices. The terms "Low," "Medium," and "High" (or $L, M$, and $H$ ) are used throughout this paper to ease exposition, but these are not the labels seen by subjects.

\footnotetext{
${ }^{9}$ Charness and Dufwenberg (2010) compare rich and limited communication in a one-shot trust game and find that rich messages makes subjects more trusting and somewhat more trustworthy. Our game is different because cooperation is consistent with equilibrium and messages about contingent strategies have an important role to play. As will be seen, our results are both stronger and have a different interpretation. Rather than revolving around differences in what can be said in the two treatments, the PIC and PChat treatments differ in the usage of a common set of messages.
} 
Subjects knew they would be playing a total of 20 rounds of the TPBG. They also knew that the first ten rounds would be played without communication and followed by a pause for additional instructions. The possibility of communication was introduced at this intermediate point. To maintain parallelism there was a pause prior to the 11th round in the $N$ treatment to announce that none of the rules would change.

In the $P 1$ and $P 1 C$ treatments, the instructions prior to round 11 described in detail what messages could be sent, including the option to send "no response," but provided no guidance about why any particular message should be sent. We stressed that the messages are cheap talk.

Subjects in the two chat treatments received extensive instructions, largely focused on the mechanics of using the chat program. The instructions gave the subjects no guidance on what types of messages should be sent other than (i) requesting that they not identify themselves and (ii) asking them to avoid offensive language. These instructions also stressed that the messages are cheap talk with no direct effect on payoffs.

Subjects had printed copies of the payoff tables for both periods available whenever they made a decision. When choosing a price for either period, the interface showed subjects any messages or chat from either player for the current period as well as a summary of outcomes (prices and payoffs) for all previous rounds. The interface automatically showed the summary for the three most recent rounds with a scroll bar that could be used to see earlier rounds. When choosing a price in Period 2, subjects could see the prices and payoffs for both players in Period 1, but could not see any communication from Period 1 . At the end of each period subjects received a summary of the prices chosen by both players as well as both players' payoffs for the period. Period 2 feedback also included the sum of payoffs across both periods for both players.

The interface (with one exception) did not include identifying information about a subjects' opponent to limit the possibility of repeated game effects across rounds. To make it possible for subjects to tell whether a message had been sent by themselves or their opponent, messages in the chat window were tagged with a randomly generated ten digit "chat id." At the time these sessions were run, we were unable to generate new chat ids across rounds or to use nonidentifying tags. Subjects were not allowed to have any writing implements during the experiment to prevent them from writing down the other players' chat ids, and it seems unlikely that they remembered long random numbers across multiple rounds. With one exception, the content of the chat contains no evidence that subjects knew when they had played an opponent previously. ${ }^{10}$ In follow-up experiments at FSU we have run sessions with both a "chat id" and nonidentifiable tags ("mine" and "other"). The type of tag has no discernible effect on messages or behavior.

Sessions were automatically ended at the two hour mark to avoid subject fatigue (this was not announced to subjects in advance). Due to this rule, one session of the $R C h a t$ treatment only had 16 rounds and another only had 18 rounds.

\footnotetext{
${ }^{10}$ One subject in the RChat treatment tried to pass on the identity of a subject who had cheated him to two other subjects (he didn't exactly remember the chat ID of the offending party, but came close). None of our conclusions change if the affected observations are dropped.
} 


\section{Results}

Collusion in the TPBG is defined in terms of Period 1 play. Any discussion of collusion in the results section refers to Period 1 choices. Our analysis of Period 2 choices focuses on how these depend on Period 1 outcomes, a central point of interest for understanding the relationship between communication and collusion. See online Appendix D for tables and figures giving frequencies of all actions in both periods for all rounds, broken down by treatment.

\section{A. Behavior in Rounds 1-10}

Initially modest levels of collusion collapse over ten rounds without communication. In Round 1, 44 percent of the subjects choose Low in Period 1 compared with only 12 percent choosing High. By Round 10, play converges to the Nash equilibrium for the Period 1 game with 86 percent of subjects choosing Low for Period 1 and only 4 percent choosing High. There is no statistically significant difference between treatments for the first ten rounds. Underlying the collapse of collusion in Rounds $1-10$ are poor incentives to collude. In Round 1 the average payoffs for the entire round (i.e., the sum of payoffs for Periods 1 and 2) following Period 1 choices of High, Medium, and Low are 34, 82, and 98 ECUs respectively. Subjects earn almost three times as much if Low is chosen in Period 1 rather than High. Similarly bad incentives exist throughout Rounds 1-10.

Period 2 choices are more diffuse than Period 1 choices, but by Round 10 a clear mode has emerged at Medium with 53 percent of all Period 2 choices. Period 2 choices are positively and significantly correlated with Period 1 choices. This effect strengthens over time, but is never sufficiently large to prevent Low from being the profit maximizing choice for Period 1. The dependence of Period 2 behavior on Period 1 outcomes suggests that the type (but not the magnitude) of contingent behavior needed for tacit collusion is present in the data before communication becomes available.

\section{B. Prices in Rounds 11-20}

Figure 1 plots the frequency of High being played in Period 1 across Rounds 10-20 for each treatment. Prior to the introduction of communication in Round 11, play of high is infrequent in all five treatments and has largely vanished by Round 10. For the $\mathrm{N}$ treatment, there is no discernible restart as play continues the pattern from Rounds $1-10$ with steady movement toward 100 percent play of Low in Period 1 . In contrast, all four communication treatments show a large initial increase in collusion as choice of High rises in Round 11 when communication becomes available. After this initial burst the evolution of play through Rounds 11-20 strongly differs across the four communication treatments.

In the two limited message space treatments, $P 1$ and $P 1 C$, an initial increase in collusion is followed by a steady collapse. The $P 1$ treatment shows the least initial increase in collusion of the communication treatments. Medium rather than High is the modal choice in Round 11. Use of High dies out over time, almost disappearing 


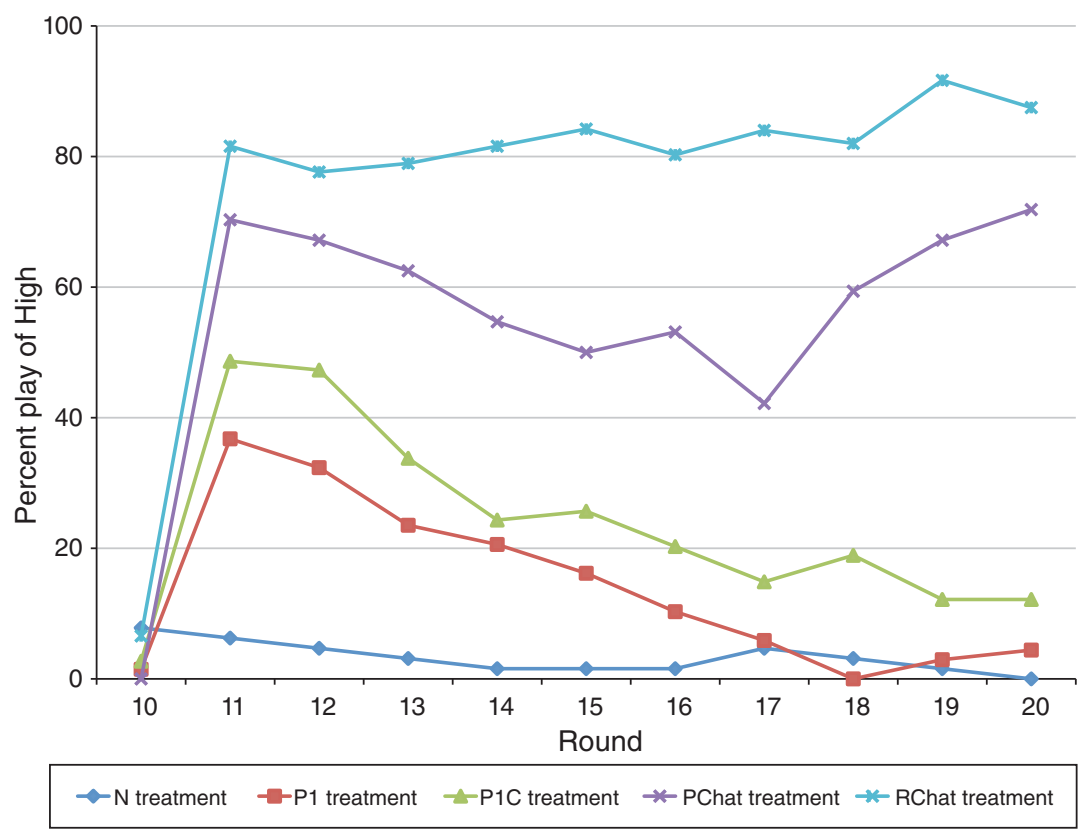

Figure 1. Play of High in Period 1

by round 20, and use of Medium drops steadily as well. Play converges toward the noncooperative outcome. The $P I C$ treatment does little better, in spite of the availability of contingent messages. The initial increase in use of High is somewhat stronger than in $P 1$, with High being the clear modal strategy in Round 11, but a similar collapse follows. Period 1 play has not converged by Round 20, but with choice of High having become quite rare (12 percent) and a mode of choosing Low (51 percent) having emerged, it is clear where things are headed.

The initial spike in play of High is much stronger in both chat treatments than in either limited message space treatment. In Round 11, the modal Period 1 choice is High for both PChat and RChat with play of Low being almost nonexistent (5 percent in both treatments). The subsequent dynamics differ between the two chat treatments. In the PChat treatment use of High falls sharply until Round 17, similar to the declines observed in the $P 1$ and $P 1 C$ treatments. Unlike the limited message space treatments this decline reverses in Rounds 17-20. Round 20 choices are largely indistinguishable from those made for Round 11 with 72 percent of the subjects choosing High. There is a clear trend towards collusion in the long run.

In the RChat treatment, no decline in collusion is observed. The distribution of Period 1 actions is essentially constant over Rounds 11-20. The small shifts upward in Rounds 17 and 19 are artifacts driven by RChat sessions ending in different rounds. If we break the data down by session, little change is observed over Rounds 11-20.

Table 2 reports the results of regressions providing statistical evidence of treatment effects in Rounds 11-20. These are ordered probit models based on all observations from Rounds 11-20. The standard errors are corrected for clustering at the individual subject level. The dependent variable is the Period 1 choice with High 
Table 2-Ordered Probit Models for Treatment Effects

\begin{tabular}{|c|c|c|c|c|c|}
\hline \multicolumn{3}{|c|}{ Comparison with no communication } & \multicolumn{3}{|c|}{ Differences between treatments } \\
\hline Variable & Estimate & Standard error & Variable & Estimate & Standard error \\
\hline P1 & $1.314 * * *$ & 0.191 & $\mathrm{P} 1-\mathrm{N}$ & $1.314^{* * * *}$ & 0.191 \\
\hline $\mathrm{P} 1 \mathrm{C}$ & $1.707 * * *$ & 0.193 & $\mathrm{P} 1 \mathrm{C}-\mathrm{P} 1$ & $0.393 * * *$ & 0.119 \\
\hline PChat & $2.651 * * *$ & 0.200 & PChat - P1C & $0.944 * * *$ & 0.122 \\
\hline RChat & $3.250 * * *$ & 0.211 & RChat - PChat & $0.599 * * *$ & 0.140 \\
\hline Pseudo $R^{2}$ & \multicolumn{2}{|c|}{0.276} & $R^{2}$ & \multicolumn{2}{|c|}{0.276} \\
\hline
\end{tabular}

Note: Standard errors have been corrected for clustering at the individual level.

*** Significant at the 1 percent level.

** Significant at the 5 percent level.

* Significant at the 10 percent level.

coded as 2, Medium coded as 1 , and Low coded as 0 . The independent variables are treatment dummies, with the $\mathrm{N}$ treatment as the base. The right panel reformulates this regression to measure differences between treatments. In all cases, the treatment effect and differences between treatments are significant at the 1 percent level. Online Appendix A reports a more sophisticated version of this analysis. Controls are included for behavior in Rounds $1-10$, and data is broken down into two round chunks so that time trends can be discussed. We find that the differences between treatments are statistically significant for (almost) all of the two round chunks. We also examine changes within treatments over time, confirming that Period 1 prices decline and then rebound significantly in the PChat treatment. The dip and recovery in the PChat treatment are unlikely to have occurred by chance.

\section{Content Analysis}

The results of the $P I$ and $P l C$ treatments show that the possibility of communicating intent to collude, even if backed with the possibility of sending Period 2 messages contingent on Period 1 outcomes, is insufficient to generate stable collusion. Something contained in the rich exchanges of the chat treatments must lead to high rates of collusion. To systematically study how the content of chat affects play we quantified content by coding all of the dialogues. The goal was to be as comprehensive as possible, including a category for any type of message that might conceivably be relevant for play of the game, rather than only coding categories we thought likely, ex ante, to be important in generating collusion. See online Appendix B for a full list of categories.

Two research assistants independently coded all messages. No effort was made to force agreement among coders and the coders were not informed about any hypotheses to be tested. Coding was binary with a message coded as a 1 if it was deemed to contain the relevant category of content and zero otherwise. We had no requirement on the number of codes for a message - a coder could check as many or few categories as he deemed appropriate.

Table 3 summarizes the frequency of the most common categories, defined as any category that was coded in at least 5 percent of the dialogues (averaging across coders). The measure of frequency reported in Table 3 is the percentage of dialogues 
TABLE 3

\begin{tabular}{|c|c|c|c|}
\hline \multicolumn{4}{|l|}{ Use of Period 1 messages in chat treatments } \\
\hline Message description & $\begin{array}{l}\text { Percent observed } \\
\text { PChat }\end{array}$ & $\begin{array}{c}\text { Percent observed } \\
\text { RChat }\end{array}$ & $\kappa$ \\
\hline Period 1 proposal: both play Medium & 21.1 & 8.1 & 0.802 \\
\hline Period 1 proposal: both play High & 88.9 & 97.5 & 0.812 \\
\hline Period 2 proposal: both play High & 93.6 & 54.1 & 0.847 \\
\hline Disagreement with most recent proposal & 10.9 & 4.0 & 0.343 \\
\hline Agreement with most recent proposal & 79.4 & 79.1 & 0.840 \\
\hline Implicit threat to punish cheating in Period 2 & 5.8 & 6.5 & 0.532 \\
\hline Explicit threat to punish cheating with Low in Period 2 & 14.1 & 1.7 & 0.755 \\
\hline Agreement with proposed punishment (all punishments) & 10.8 & 2.3 & 0.451 \\
\hline Request for proposals & 7.7 & 11.8 & 0.721 \\
\hline Appeal to mutual benefits & 29.7 & 15.7 & 0.587 \\
\hline Reference to safety or risk of strategies & 7.5 & 2.5 & 0.397 \\
\hline Specific reference to payoff table & 16.9 & 9.6 & 0.694 \\
\hline Promises of trustworthy behavior & 11.1 & 8.6 & 0.624 \\
\hline Expression of distrust & 11.1 & 3.6 & 0.448 \\
\hline Appeal for trustworthy behavior & 15.3 & 6.8 & 0.460 \\
\hline Self-report having been cheated in earlier rounds & 16.9 & 10.8 & 0.812 \\
\hline \multicolumn{4}{|l|}{ Use of Period 2 messages in RChat treatment } \\
\hline & Percent observed & Percent observed & \\
\hline Message description & Period 1 , collusion & Period 1 , cheated & $\kappa$ \\
\hline Positive feedback following cooperation & 28.3 & 2.3 & 0.731 \\
\hline Apology for cheating & - & 47.7 & 0.778 \\
\hline Rationalizing cheating & - & 44.7 & 0.671 \\
\hline Admonition for cheating/lying & - & 56.8 & 0.623 \\
\hline Period 2 proposal: both play High & 90.1 & 89.4 & 0.792 \\
\hline Agreement with most recent proposal & 67.5 & 39.4 & 0.473 \\
\hline Appeal to mutual benefits & 1.7 & 26.5 & 0.366 \\
\hline
\end{tabular}

where the category was coded. For messages prior to Period 2 from the $R C h a t$ treatment, observations are broken down by whether both players colluded in Period 1 (both choose High) or one player cheated (deviated from an agreement to choose High). Observations where both players cheated are not included. The final column of each panel shows $\kappa$, a common measure of inter-coder agreement (Cohen 1960). The $\kappa$ 's generally show substantial agreement between the coders, especially since the number of possible codes was large and no attempt was made to force agreement between coders.

Subjects almost always engaged in communication relevant to the game. Period 1 prices were proposed in 618 of 622 dialogues, and only one dialogue lacked substantive discussion of how the game would be played. The content of the dialogues differed substantially between the PChat and RChat treatments, and there was generally more substantive conversation prior to Period 1 in the PChat treatment. Since subjects both behave differently in the two chat treatments and communicate differently, we do not pool data from the two chat treatments in the content analysis.

Agreements and Cheating.- - In all four communication treatments, we can identify agreements between the players on what price to set in Period 1. For the P1 and $P I C$ treatments, we define the players as having come to an agreement if their messages prior to Period 1 suggest the same Period 1 prices. In the PChat and RChat 


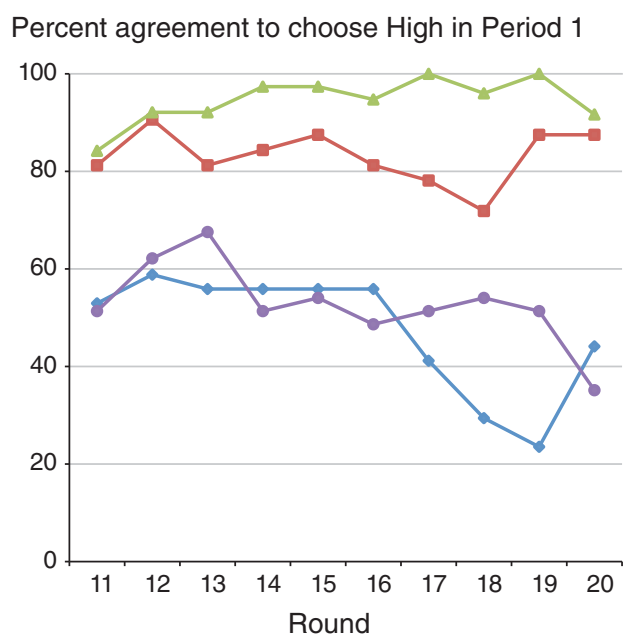

$\rightarrow \mathrm{P} 1$ treatment $\rightarrow \mathrm{P} 1 \mathrm{C}$ treatment

- PChat treatment - RChat treatment
Percent cheating on an agreement to choose High in Period 1

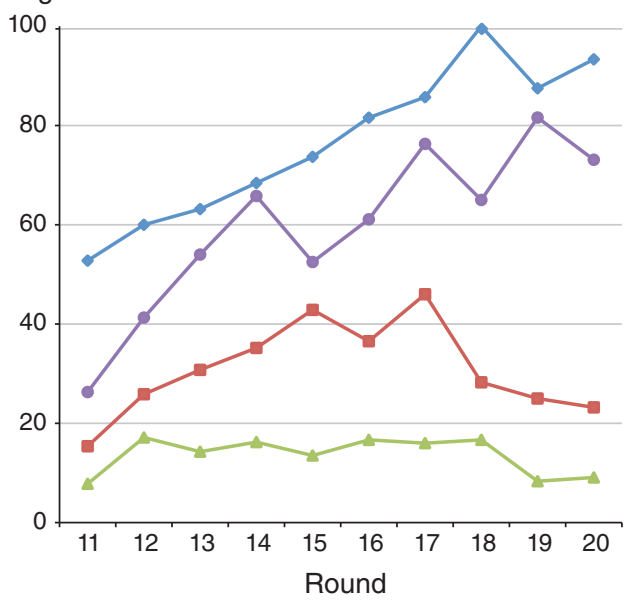

$$
\begin{aligned}
& \leftarrow \text { P1 treatment } \quad \leftarrow \text { P1C treatment } \\
& - \text { PChat treatment } \quad \leftarrow \text { RChat treatment }
\end{aligned}
$$

Figure 2. Agreements And Cheating

treatments, the players are defined as having an agreement if a proposal for Period 1 prices is made by one player and then accepted by the other.

The left panel of Figure 2 shows the percentage of pairs agreeing to play High in Period 1, broken down by round and treatment. Pairs almost always reach an agreement on Period 1 prices in the PChat (98 percent reach agreement) and $R$ Chat treatments (100 percent reach agreement), but often do not reach agreements in the $P 1$ (51 percent reach agreement) and $P I C$ treatments (55 percent reach agreements). It is relatively hard to reach an agreement in the limited message space treatments since subjects cannot revise proposals when their initial proposals do not agree. Subject to reaching an agreement, players in all treatments with communication overwhelmingly agree on choosing High in Period 1 (92 percent in $P 1,97$ percent in $P 1 C, 85$ percent in PChat, and 94 percent in $R C h a t)$.

We define cheating as choosing Low or Medium in Period 1 following an agreement to choose High in Period 1. The right panel of Figure 2 shows the frequency of cheating broken down by round and treatment. The probability of cheating in the $P 1$ and $P I C$ treatments is substantial even in early rounds and rises over time. The high frequency of cheating indicates that the cause of declining collusion in the $P 1$ and $P I C$ treatments is not a failure to reach agreements on play of High in Period 1. Even if all pairs reached collusive agreements, it is unlikely that collusion could survive such pervasive cheating.

For the chat treatments, cheating follows a matching pattern to that observed for play of High in Figure 1. Cheating in the PChat treatment is initially only slightly higher than in the RChat treatment, but rises steadily to a peak in Round 17 . It then declines back to almost its initial level. Cheating in the RChat treatment is low and steady throughout. 
The standard theory of collusion suggests that the decision to cheat on an agreement to collude is driven by whether cheating is likely to be punished. Can we then explain the low levels of cheating in the two chat treatments by relatively high likelihood of punishment?

Define unilateral cheating as the case where one player cheats on an agreement to choose High in Period 1 while the other player does not. Unilateral cheating is frequently punished in Rounds 11-20 of all four treatments with communication. Punishment for unilateral cheating is relatively strong in the two treatments with limited message spaces. In the $P l$ treatment, the proportion of noncheaters (subjects who do not cheat on an agreement to choose High) choosing High in Period 2 drops from 90 percent when no cheating occurs to 9 percent when the opponent cheats. In the $P 1 C$ treatment this proportion goes from 99 percent to 33 percent. Punishment is weaker in the two chat treatments. The proportion of noncheaters that choose High in Period 2 changes from 97 percent to 45 percent when their opponent cheats in the PChat and from to 100 percent and 65 percent in the RChat treatment. Punishment of cheating is significantly weaker in the RChat treatment than in the PChat treatment, consistent with the theory of renegotiation, and the difference between treatments grows over time. ${ }^{11}$ Differing frequencies of punishment do not explain the relatively low levels of cheating in the two chat treatments or the low level of cheating in the RChat treatment relative to the PChat treatment.

Of course, the frequency of punishment is not the only factor determining incentives to cheat, as the payoff from cheating depend both on whether unilateral cheating will be punished and the likelihood that the other player will cheat. There are strong incentives to cheat in $P 1$ and $P l C$ because of frequent cheating by others. This becomes obvious when comparing total payoffs for the round (summing over both periods) when a subject cheats on a collusive agreement with the total payoffs when he does not cheat. In the $P 1$ and $P 1 C$ treatments, the average increases in total payoff from cheating are 19 and 26 ECUs respectively in Rounds 11-12, increasing to 36 and 43 ECUs for Rounds 19-20. As a point of comparison, a subject gains 54 ECUs by cheating on a collusive agreement if the other player does not cheat and Period 2 actions are unaffected.

The incentives to cheat in the PChat treatment are initially large, primarily because of the relatively low threat of punishment. The average increase in total payoff from cheating on an agreement is 31 ECUs in Rounds 11-12. Over time the incentive to cheat shrinks to an average of 12 ECUs in Rounds 19-20. This reflects both an increase in punishment and a decreasing threat over Rounds 17-20 of being cheated. The incentives to cheat are in line with the initial decrease in collusion, but do not fully explain why collusion recovers in later rounds since cheating continues to pay (albeit, by less). The opposite trend on the incentives to cheat emerges in the RChat treatment. The average gain from cheating is only 10 ECUs over Rounds 11-12, but it jumps to 35 ECUs for Rounds 15-16 (the last two rounds before the

\footnotetext{
${ }^{11}$ In Rounds 16-20 unilateral cheating lowers the proportion of Period 2 choices of High by noncheaters from 98 percent to 39 percent in PChat versus a change from 100 percent to 75 percent in RChat. Punishment is significantly more likely in PChat at the 5 percent level for Rounds 11-20 and the 1 percent level for Rounds 16-20 based on the results of ordered probit regressions controlling for round effects and individual effects. This is based on 99 observations for PChat and 65 observations for RChat.
} 
first session ended). There is little danger of getting cheated in the RChat treatment, but punishment is sufficiently weak in the later rounds that compliance with a collusive agreement is not incentive compatible. Nonetheless, collusion remains stable.

The analysis of cheating and punishment leaves us with a pair of puzzling observations. In neither chat treatment are the average gains from cheating negative at any point, yet both treatments show high levels of collusion. Likewise, the incentives to cheat are stronger in the long run for the RChat treatment than the PChat treatment, yet this is the treatment with the highest and most stable levels of collusion. These observations only make sense if something in the message content counteracts the generally poor incentives to honor collusive agreements. We therefore turn to the effects of specific types of messages.

Threats and Promises.-An agreement to play High is only credible if accompanied by a threat that failure to cooperate in Period 1 will be punished by play of Low. Our coding scheme distinguishes between two different types of threats to punish cheating, explicit and implicit. An explicit threat of punishment refers to cases where subjects specifically state that failure to collude will lead to use of Low rather than High in Period 2. An implicit threat of punishment refers to cases where a subject threatened to punish noncollusion but did not specify what strategy would be used in Period 2. The top panel of Figure 3 shows the frequency of explicit and implicit threats (averaged across the two coders) in the two chat treatments.

The use of threats rises over time in both treatments. The frequency of threats is always higher in the PChat treatment than in the RChat treatment, and most threats are explicit in the PChat treatment while most threats in the RChat treatment are implicit. ${ }^{12}$

The theories of collusion and cheap talk make threats of punishment a natural focus for our analysis of chat content, but the psychology and economics literatures both suggest that promises could play an important role in increasing cooperation (Kerr and Kaufman-Gilliland 1994; Charness and Dufwenberg 2006). Communication can also reinforce group identity and norms of maximizing joint benefits (Dawes, Van De Kragt, and Orbell 1988), providing yet another possible explanation for decreased cheating in the last few rounds of the PChat treatment.

Three common coding categories relate to these alternative explanations for the positive effect of chat: promises of trustworthy behavior, ${ }^{13}$ appeals for trustworthy behavior by the other player, and appeals to the mutual benefits of collusion. The bottom panel of Figure 3 shows the percentage of dialogues (averaged across coders) in the PChat and RChat treatment where these three categories were observed. In both treatments, appeals to the mutual benefits of collusion are consistently more common than either promises of trustworthy behavior, appeals for trustworthy behavior,

\footnotetext{
${ }^{12}$ The difference in frequency of threats (explicit or implicit) between the PChat and RChat treatments is significant at the 5 percent level in Rounds 16-20. For explicit threats, the difference is significant at the 10 percent level for Rounds 11-15 and at the 1 percent level for Rounds 16-20. These statements are based on ordered probits with round dummies (not interacted with the treatment dummy) as controls and standard errors corrected for clustering at the subject level.

${ }^{13}$ This category also includes messages where subjects indicate that they should be trusted (e.g., "uve just gotta trust me" and "well, just trust me").
} 

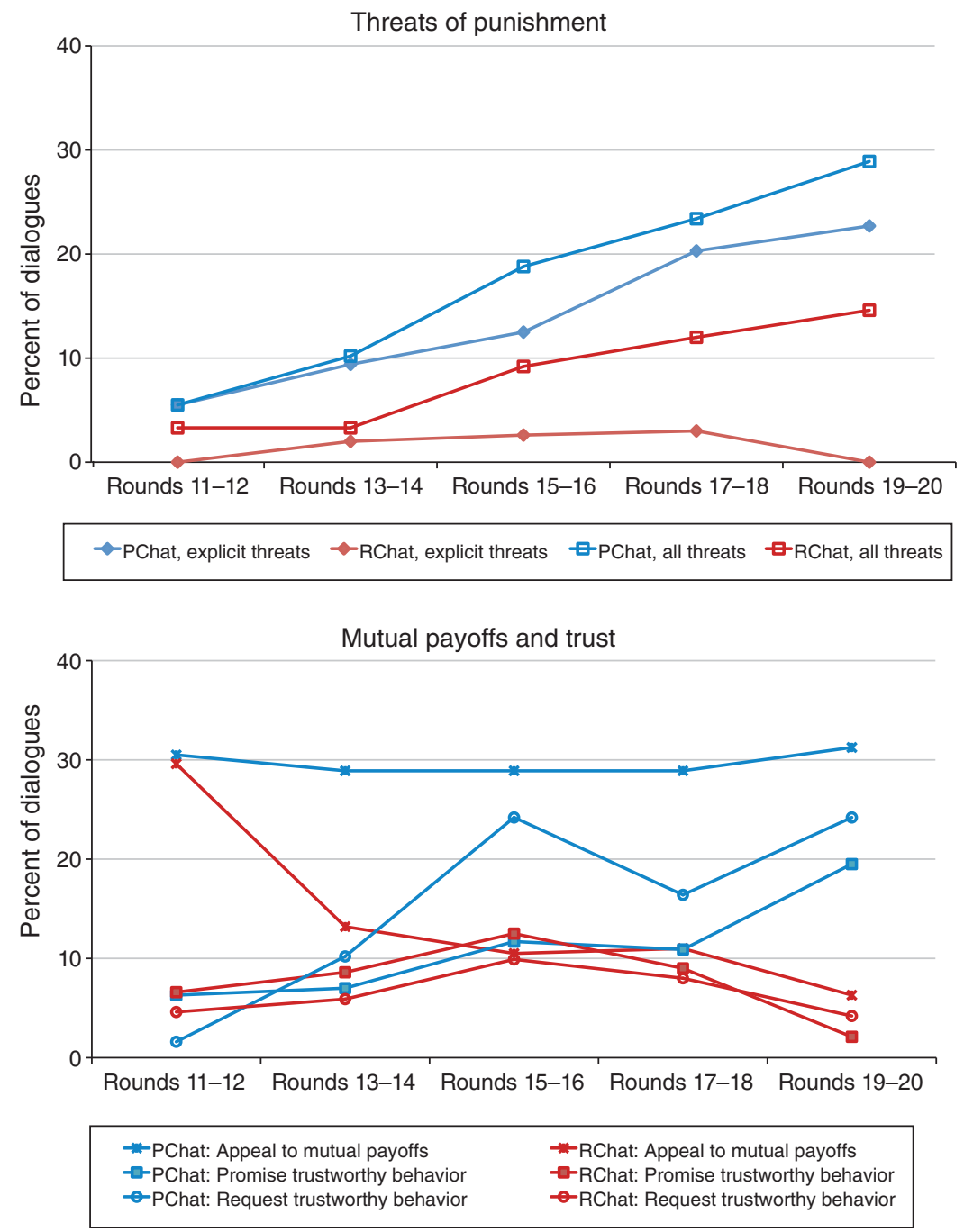

Figure 3. Frequency of Chat Categories over Time

or explicit threats. In the PChat treatment, coding of requests for and promises of trustworthy behavior become more common over time, but these categories remain rare throughout the RChat treatments. Appeals to the mutual benefits of collusion also drop off sharply over time in the RChat treatment.

It is nontrivial to sort out the effects of the different message categories. A naïve approach is to compare the level of cheating in observations where a message category is coded with the frequency of cheating when the category is not coded. In the PChat treatment, for example, the likelihood of cheating is lower when either an explicit threat is observed (10 percent with versus 35 percent without) or, to a lesser extent, an appeal to the mutual benefits of collusion ( 25 percent with versus 34 percent without). Unfortunately, these observations do not necessarily capture a causal relationship between the categories in question and cheating. At the very least we need to distinguish between whether a subject sent or received the 
message in question. Causal statements only make sense in the latter case. Even after accounting for this, a major problem remains because most dialogues include many codes, including codes that we have not thus far discussed. Since the coding of categories is often correlated, comparisons of cheating with and without a category being coded may reflect a failure to control for the effects of other types of messages. To address these issues we turn to regression analysis.

Table 4 reports the results for regressions in which the dependent variable is a dummy for whether the subject cheated on an agreement. Observations where an agreement was not reached ( 1 percent of the dataset) are dropped. Cheating is a binary variable, so the regressions use a probit specification. Standard errors (in parentheses) are corrected for clustering at the subject level. We report both parameter estimates (before the slash) and marginal effects (after the slash) for each variable.

The independent variables of primary interest measure whether a comment from a specific category was received or sent by the individual in the dialogue prior to Period 1. These variables are averages across the two coders and therefore have three possible values: $0, \frac{1}{2}$, and 1 . With a few exceptions, the regressions incorporate all categories that were coded in at least 5 percent of dialogues. ${ }^{14}$ As additional controls, the regressions include dummies for the agreed upon price, the current round, the subject's average Period 1 price in Rounds $1-10$, and the average Period 1 price of their opponent in Rounds 1-10. The subject's own history of cooperation in Rounds 1-10 provides a control for unobserved individual effects beyond the correction for clustering. The inclusion of the opponent's history of cooperation in Rounds 1-10 controls for a potential source of omitted variable bias. The opponent's history cannot have a direct effect on decisions to cheat, since subjects do not know their opponent's history, but individuals who are inherently more cooperative may also communicate in subtly different ways not captured by the coding.

Omitted variable bias can also arise due to the interactive nature of dialogues. There is correlation between the types of messages that are sent and the types that are received. This can make it appear that receipt of a message causes cooperative action, when the effect is actually driven by a related message the subject sent. Including controls for sent messages eliminates this bias as the impact of sent messages is directly accounted for. As it turns out, none of our conclusions about the effects of receiving messages change with controls for sent messages.

The parameter estimates for the sent messages should be interpreted as identifying associations rather than causal relationships with cheating. Because a strategy for sending messages and choosing prices could be jointly determined prior to the beginning of a round, it is impossible to identify a causal relationship between them.

The regression was run separately on the PChat and RChat data. The parameter estimates for the agreement dummies, round dummies, and controls for Period 1 prices in Rounds 1-10 are not of direct interest and are not reported in Table 4 to save space. Looking at the effect of received messages in the PChat data, only

\footnotetext{
${ }^{14}$ Because we include dummies for the agreed upon price as independent variables, we do not include the messages proposing prices to avoid colinearity. Likewise, the category for agreeing to a punishment scheme is highly correlated with sending a threat, and is excluded to avoid colinearity.
} 
Table 4-Probit Regressions on Effect of Chat Categories

\begin{tabular}{|c|c|c|}
\hline & PChat & RChat \\
\hline Number of observations/subjects & $626 / 64$ & $602 / 76$ \\
\hline $\begin{array}{l}\text { Received } \\
\text { Implicit threat }\end{array}$ & $\begin{array}{l}0.475 / 0.152 \\
\quad(0.453)\end{array}$ & $\begin{array}{c}-1.211 /-0.251^{* *} \\
(0.503)\end{array}$ \\
\hline $\begin{array}{l}\text { Received } \\
\text { Explicit threat }\end{array}$ & $\begin{array}{c}-0.808 /-0.259 * * * \\
(0.310)\end{array}$ & $\begin{array}{l}0.556 / 0.115 \\
(0.875)\end{array}$ \\
\hline $\begin{array}{l}\text { Received } \\
\text { Request for proposals }\end{array}$ & $\begin{array}{c}0.215 / 0.069 \\
(0.382)\end{array}$ & $\begin{array}{l}0.370 / 0.077 \\
\quad(0.292)\end{array}$ \\
\hline $\begin{array}{l}\text { Received } \\
\text { Appeal to mutual payoffs }\end{array}$ & $\begin{array}{c}-0.284 /-0.091 \\
(0.194)\end{array}$ & $\begin{array}{c}-0.022 /-0.004 \\
(0.294)\end{array}$ \\
\hline $\begin{array}{l}\text { Received } \\
\text { Reference to safety or risk of strategies }\end{array}$ & $\begin{array}{c}-0.101 /-0.032 \\
(0.403)\end{array}$ & $\begin{array}{c}0.802 / 0.166 \\
(0.624)\end{array}$ \\
\hline $\begin{array}{l}\text { Received } \\
\text { Specific reference to payoff table }\end{array}$ & $\begin{array}{c}-0.119 /-0.038 \\
(0.269)\end{array}$ & $\begin{array}{c}0.128 / 0.026 \\
(0.331)\end{array}$ \\
\hline $\begin{array}{l}\text { Received } \\
\text { Promise of trustworthy behavior }\end{array}$ & $\begin{array}{c}-0.167 /-0.054 \\
(0.292)\end{array}$ & $\begin{array}{c}-0.079 /-0.016 \\
(0.617)\end{array}$ \\
\hline $\begin{array}{l}\text { Received } \\
\text { Expression of distrust }\end{array}$ & $\begin{array}{l}0.206 / 0.066 \\
\quad(0.285)\end{array}$ & $\begin{array}{l}1.632 / 0.338^{* *} \\
\quad(0.830)\end{array}$ \\
\hline $\begin{array}{l}\text { Received } \\
\text { Appeal for trustworthy behavior }\end{array}$ & $\begin{array}{c}0.369 / 0.118 \\
(0.258)\end{array}$ & $\begin{array}{c}-0.256 /-0.053 \\
(0.633)\end{array}$ \\
\hline $\begin{array}{l}\text { Received } \\
\text { Self-report being cheated earlier }\end{array}$ & $\begin{array}{c}-0.175 /-0.056 \\
(0.231)\end{array}$ & $\begin{array}{c}0.232 / 0.048 \\
(0.298)\end{array}$ \\
\hline $\begin{array}{l}\text { Sent } \\
\text { Implicit threat }\end{array}$ & $\begin{array}{c}-0.419 /-0.134 \\
(0.474)\end{array}$ & $\begin{array}{l}\text { Perfectly predicts } \\
\text { no cheating }\end{array}$ \\
\hline $\begin{array}{l}\text { Sent } \\
\text { Explicit threat }\end{array}$ & $\begin{array}{c}-1.261 /-0.404 * * * \\
(0.371)\end{array}$ & $\begin{array}{l}\text { Perfectly predicts } \\
\text { no cheating }\end{array}$ \\
\hline $\begin{array}{l}\text { Sent } \\
\text { Request for proposals }\end{array}$ & $\begin{array}{l}-0.284 /-0.091 \\
(0.425)\end{array}$ & $\begin{array}{c}0.235 / 0.049 \\
(0.316)\end{array}$ \\
\hline $\begin{array}{l}\text { Sent } \\
\text { Appeal to mutual payoffs }\end{array}$ & $\begin{array}{c}-0.223 /-0.071 \\
(0.247)\end{array}$ & $\begin{array}{c}-0.430 /-0.089 \\
(0.371)\end{array}$ \\
\hline $\begin{array}{l}\text { Sent } \\
\text { Reference to safety or risk of strategies }\end{array}$ & $\begin{array}{c}-0.053 /-0.017 \\
(0.427)\end{array}$ & $\begin{array}{l}0.897 / 0.186^{*} \\
\quad(0.469)\end{array}$ \\
\hline $\begin{array}{l}\text { Sent } \\
\text { Specific reference to payoff table }\end{array}$ & $\begin{array}{c}0.257 / 0.082 \\
(0.287)\end{array}$ & $\begin{array}{l}0.573 / 0.119 \\
\quad(0.407)\end{array}$ \\
\hline $\begin{array}{l}\text { Sent } \\
\text { Promise of trustworthy behavior }\end{array}$ & $\begin{array}{c}-0.603 /-0.193 * * \\
(0.243)\end{array}$ & $\begin{array}{c}-1.686 /-0.349 * * * \\
(0.486)\end{array}$ \\
\hline $\begin{array}{l}\text { Sent } \\
\text { Expresion of distrust }\end{array}$ & $\begin{array}{l}0.316 / 0.101 \\
(0.383)\end{array}$ & $\begin{array}{c}-0.580 /-0.120 \\
(1.109)\end{array}$ \\
\hline $\begin{array}{l}\text { Sent } \\
\text { Appeal for trustworthy behavior }\end{array}$ & $\begin{array}{c}-0.455 /-0.146 \\
(0.379)\end{array}$ & $\begin{array}{c}0.298 / 0.062 \\
(0.678)\end{array}$ \\
\hline $\begin{array}{l}\text { Sent } \\
\text { Self-report being cheated earlier }\end{array}$ & $\begin{array}{c}-0.281 /-0.090 \\
(0.241)\end{array}$ & $\begin{array}{c}-0.408 /-0.085 \\
(0.316)\end{array}$ \\
\hline $\log$ likelihood & -325.26 & -221.58 \\
\hline Pseudo $R^{2}$ & 0.125 & 0.070 \\
\hline
\end{tabular}

*** Significant at the 1 percent level.

** Significant at the 5 percent level.

* Significant at the 10 percent level.

explicit threats have a significant effect on the likelihood of cheating. The marginal effect is large, with an estimated 26 percent reduction in the probability of cheating. Receiving an appeal to the mutual benefits of collusion, a promise of trustworthy behavior, or an appeal for trustworthy behavior all fail to have a significant effect 
on the likelihood of cheating. The PChat regression shows that explicit threats are by far the type of message whose receipt has the strongest effect on collusion in Period 1. In the Appendix we explore a wide variety of alternative specifications and find that this result is quite robust.

Turning to sent messages, subjects who send explicit threats cheat significantly less. The marginal effect is large, with an estimated 40 percent reduction in cheating. Subjects who promise to be trustworthy are also significantly less likely to cheat on an agreement. The marginal effect is large at 19 percent, but less than half of the marginal effect of sending an explicit threat.

The RChat regression shows that Period 1 communication has a somewhat different impact in the RChat treatment than in the PChat Treatment. Receiving an implicit threat significantly reduces cheating, while receiving an explicit threat has no significant effect. The estimated marginal effect from receiving an implicit threat in the RChat treatment is about the same as the marginal effect of an explicit threat in the PChat treatment (25 percent versus 26 percent). Receiving an expression of distrust significantly increases cheating. This makes sense, but should be interpreted cautiously given the rarity of such comments.

Sending either an implicit or explicit threat has a significant negative effect on cheating. Sending a promise of trustworthy behavior once again has a strong negative effect on cheating. There is a positive effect on cheating from sending a message referring to the safety or risk of strategies, but this is only weakly significant.

Threats do not explain the initial jump in collusion for the PChat treatment. When a subject receives an explicit threat in Rounds 11-12 of the PChat treatment (the first two rounds with communication), the subject never cheats on a collusive agreement. This sounds impressive, but there are only four such observations. The importance of threats emerges over time as this type of comment becomes more frequent, providing an explanation for why collusion returns to its initial levels rather than collapsing as in $P l$ and $P l C$. Similar comments apply to the RChat treatment. Implicit threats have the expected effect on cheating in Rounds 11-12, but are too rare to explain the initial high levels of collusion.

Econometric analysis like that reported in Table 4 fails to find a relationship between either appeals to mutual benefits or promises of trustworthy behavior and cheating in Rounds 11-12 of the PChat treatment. Reaching an agreement to collude has a significant effect on whether collusion occurs. As can be seen in Figure 2, such agreements are more common in Rounds 11-12 of PChat as compared to PlC. This provides a partial explanation for by the higher frequency of collusion in early rounds.

Chat and the Incentives to Cheat.-We now can resolve the puzzles that subjects in the chat treatments cheat less than expected given the weak incentives to collude and that collusion rebounds in the last periods of the PChat treatment. The key insight is that what matters to subjects is not the average incentive to cheat, but instead the incentive to cheat given the specific messages they have sent and received.

For each case where we found a statistically significant reduction of cheating from sending and/or receiving a type of message, Figure 4 displays the relationship 

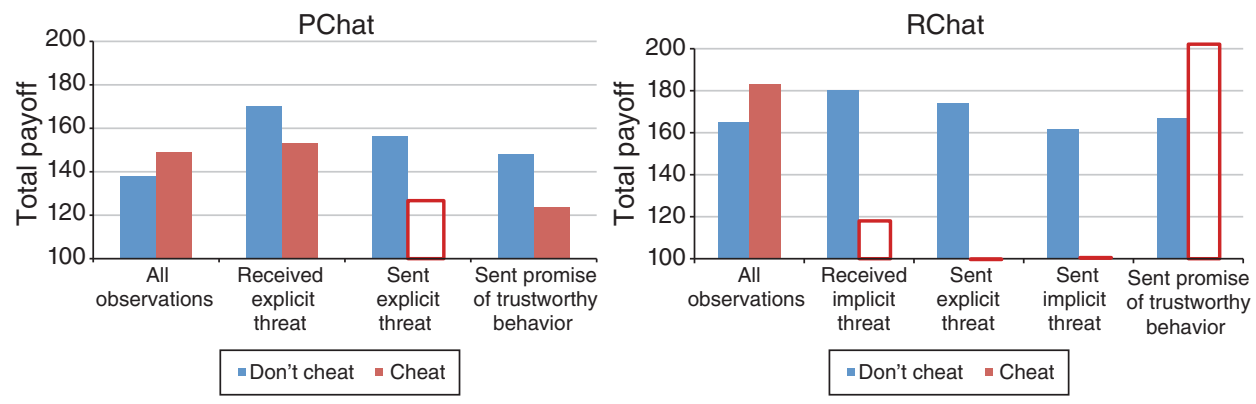

Figure 4. GaINS From CheATING AND CHAT

between sending and/or receiving this type of message and the incentives to cheat. A message is categorized as including a category if this was coded by either coder. Data is only included from observations where the subjects agreed to play High in Period 1. The average total payoffs for the entire round are plotted conditional on whether or not the player cheated on the agreement. Hollow bars indicate cells with less than five observations. Two cells had no observations and therefore are missing. As a point of comparison we also plot the corresponding average total payoff for all observations with agreements to play High in Period 1.

Types of communication that reduce cheating generally reduce the incentives to cheat. The cumulative effect of messages associated with significantly lower cheating on the incentives to cheat is large. In the PChat treatment, subjects who receive an explicit threat, send an explicit threat, or send a promise to not cheat significantly lower their average payoff by cheating on a collusive agreement $(\Delta=-27.7$ ECUs, $t=2.27, p<0.05)$ compared to a significant average gain from cheating otherwise $(\Delta=23.5$ ECUs, $t=3.18, p<0.01)$. In line with these incentives, there is only a 14 percent chance of cheating by subjects who receive an explicit threat, send an explicit threat, or send a promise as compared with 36 percent otherwise. Over time the likelihood of effective messages strongly increases, creating the conditions for a return to collusion.

We cannot say much about what happens following cheating in the RChat treatment due to a paucity of observations. Subjects who do not receive an explicit threat, send an implicit or explicit threat, or send a promise of trustworthy behavior significantly increase their payoffs by cheating $(\Delta=19.0$ ECUs, $t=2.94, p<0.01)$. There is no significant effect from cheating for subjects who do receive or send such messages, but this says little since there are only three observations with cheating in this subset.

PIC versus PChat. - In the PChat treatment, explicit threats play a central role in fostering collusion. Threats to punish cheating with Period 2 play of Low are also available in the $P l C$ treatment, yet collusion shows no sign of recovering over time in this treatment. The reason is not obvious. It should be easier for subjects to learn to use threats of punishment in the $P l C$ treatment, since the design suggests the possibility of contingent strategies to the subjects and there are no other types of messages to distract them. Indeed, explicit threats of punishment for deviating 
from a collusive agreement are more common in the $P 1 C$ treatment (24 percent of pairs) than in the PChat treatment (18 percent of pairs). The cause of lower collusion in $P I C$ also does not seem to be that the single round of communication makes it more difficult to come to an agreement than in PChat. In follow up experiments where subjects could take multiple sequential turns sending messages, agreement rates were much higher than in the original $P 1 C$ treatment but collusion was no more frequent (Cooper and Kühn 2013). The difference between the PIC and PChat treatments could be purely an effect of the differing mediums of communication (see Brosig, Ockenfels, and Weimann 2003), but the specifics of communication in the two treatments suggest otherwise.

The PIC and PChat treatments differ in the types of explicit punishments proposed. In the PChat treatment, all messages coded as explicit threats call for play of Low in Period 2 if the other player cheats. Subjects never threaten to punish cheating with play of Medium. In contrast, 90 percent of explicit threats in the PlC treatment call for cheating to be punished with play of Medium in Period 2. Subjects in the PIC treatment therefore almost never use threats which would make collusion incentive compatible. For subjects who receive an explicit threat, the average loss from cheating on an agreement is negligible (0.3 ECUs). Not surprisingly given these weak incentives, receiving an explicit threat makes subjects in the $P 1 C$ treatment no more likely to abide by a collusive agreement: 65 percent of subjects receiving explicit threats cheat on collusive agreements versus 56 percent for all others. Unlike the PChat treatment, explicit threats are ineffective in the PIC treatment, subjects abandon their use, and collusion vanishes over time.

Communication is fundamentally different when subjects participate in a natural conversation rather than using a limited message space. This is not just because more types of messages are available and these can be combined and sequenced in more ways. Our subjects use the available messages less effectively with a limited message space than when messages are embedded within the natural environment of a conversation.

The Effect of Renegotiation.-Effective messages prior to Period 1 improve the local incentives to collude, but these improved incentives do not provide a satisfactory explanation for consistently high collusion in the RChat treatment. Effective messages are uncommon until the later rounds but collusion is high and stable throughout. Moreover, cheating is rare (16 percent) even when effective messages are not present. It is also puzzling that implicit threats are effective in the RChat treatment given that they are not in the PChat treatment and the theory of cheap talk gives no reason why nonspecific threats should be useful.

The obvious difference between the PChat and RChat treatments is the addition of a communication phase between the Period 1 and 2 decisions. Consistent with the theory of renegotiation, attempts at renegotiation following unilateral cheating are frequent and reasonably successful. For games with unilateral cheating on collusive agreements, mutual play of High in Period 2 is suggested by at least one of the subjects in 89 percent of the Period 2 dialogues. When such suggestions occur, 64 percent of the pairs successfully coordinate on mutual play of High in Period 2. This compares to 14 percent of pairs coordinating on mutual play of High in Period 2 for 
the (admittedly infrequent) cases where play of High is not suggested. Successful renegotiation causes the financial consequences of cheating to be weak in the RChat treatment, creating strong incentives to cheat in Period 1

A critical point missing from the theory of renegotiation is that communication after a deviation allows cheated subjects to engage in verbal punishments. Negative verbal responses to cheating are quite common in our data. In cases of unilateral cheating on a collusive agreement, 73 percent of the subjects who were cheated admonished the other player for cheating and/or lying. The messages were often quite harsh (and ignored the instructions to avoid cursing). Examples include "good job, [expletive deleted]," "you are a bad person ... i hope someone [expletive deleted] you over as well," and (our favorite) "you know, they shoot you for that in Texas." Messages of this type are best interpreted as nonpecuniary punishments in the spirit of Masclet et al. (2003). Verbal punishment provides a cheap way to punish cheating..$^{15}$ If subjects dislike being told off, we would expect less cheating than when only conventional punishment (choosing Low in Period 2) is possible.

While the subjects who admonish cheating presumably believe they are punishing the recipients, the recipients may not care. There is a sharp difference in behavior between those subjects who never received an admonishment and those who ever received an admonishment. For convenience, we label these groups as "noncheaters" and "cheaters." Most of the subjects (56/76) are noncheaters. Noncheaters only cheat on 2 percent of collusive agreements and frequently use verbal punishment, admonishing cheating/lying in 80 percent of observations where they followed a collusive agreement and their opponent cheated. We cannot provide direct evidence that noncheaters dislike receiving verbal punishment since they do not discuss the matter and, by definition, never receive verbal punishment. However, their own frequent use of verbal punishment suggests they think receiving verbal punishment is bad. The effectiveness of implicit threats, which may be seen as threats of nonpecuniary punishment, and the low usage of explicit threats can also be seen as indirect evidence that receiving verbal punishments is considered bad.

Cheaters, in contrast, cheat on 49 percent of collusive agreements, with the probability of cheating remaining stable over time. In 80 percent of the observations in which cheaters cheat, they are admonished. If they disliked getting admonished, we would expect them to cheat less following an admonishment. Instead, the rate of cheating by cheaters who have previously been admonished rises slightly to 52 percent. When cheaters do not cheat but get cheated by their opponents, they are far less likely to admonish (42 percent) than noncheaters.

Our population thus appears to consist of two types with relatively stable (if very different) behavior. Stable cooperation occurs in the RChat treatment because the vast majority of our subjects were noncheaters who use verbal punishment and presumably fear receiving it. We can make an educated guess that noncheaters do not cheat, in spite of poor monetary incentives, to avoid verbal punishment. Cheaters

\footnotetext{
${ }^{15}$ Verbal punishment is often combined with conventional punishment. Subjects who were cheated and sent a verbal punishment were also more likely to engage in conventional punishment: 42 percent of subjects who were cheated and admonished their opponents chose a price other than High, compared with only 22 percent of subjects who were cheated and did not admonish their opponent.
} 
do not seem to be inhibited by verbal punishment, but there are too few of them to destabilize the social norm of cooperation

\section{Conclusions}

The primary purpose of our experiment was to study how communication facilitates the development of stable collusion. Our results indicate that allowing subjects to communicate an intent to collude is insufficient to generate persistent collusion even if subjects can send contingent messages calling for punishment of cheating, but allowing a rich message space leads to persistent collusion. As suggested by theory, the use of explicit threats to punish deviation from collusive agreements is the most effective type of message for promoting collusion when only pregame communication is allowed. Collusion is also promoted by sending a message promising trustworthy behavior. In sessions where renegotiation is allowed, high levels of collusion occur contrary to standard theories of renegotiation. While attempts at renegotiation occur and are reasonably successful, as predicted by theory, the effect of renegotiation is reversed by the impact of verbal punishment of cheating which provides an inexpensive and easily understood means of supporting collusion. Preplay use of implicit threats is quite effective in the renegotiation treatment, presumably because implicit threats raise the specter that cheating will be met by verbal punishment.

In our experiments, monetary incentives combine with behavioral factors to explain collusive behavior. In the PChat treatment, explicit threats cannot explain the initial increase in collusion (indeed no type of message beyond agreements to collude explains the high rates of collusion in early rounds), but play a central role in the persistence of collusion. Sending promises of trustworthy behavior leads to reduced cheating, in line with the results of Charness and Dufwenberg (2006). A plausible explanation for this result is that subjects either would feel guilty about breaking a promise or letting down their opponent (lie aversion and guilt aversion to use current jargon). However, as can be seen in Figure 4, lying about trustworthy behavior is financially punished in the PChat treatment (in the very small number of observations where it occurs). Leaving aside subtle psychological factors like guilt aversion, subjects may have a straightforward pecuniary reason not to renege on promises. Neither receiving a promise of trustworthy behavior nor an appeal to mutual payoffs has a significant effect on the likelihood of cheating. We therefore have little direct evidence that communication is successfully building trust between opponents or reducing social distance.

The most surprising result of our paper is that renegotiation facilitates collusion even though the basic logic of renegotiation theory finds support in the data. A natural question to ask is whether a result that relies so heavily on emotional reactions from subjects extends to other settings, particularly decisions made in a corporate setting. We suspect that this depends on several factors. First, our result depends on the frequency of two distinct types in our population, noncheaters and cheaters. There is no reason to assume, ex ante, that the mix of types is fixed across populations and is especially likely to vary in corporate settings where strong selection processes are in play. Thus, although we expect the same basic forces and types of 
individuals to be present in most populations, they need not balance in such a way that cooperation is higher with renegotiation. Second, differences between settings can occur due to the differing social context. As an example, most people would agree that lying is immoral, but do not feel that this rule applies when they are playing poker. If subjects perceive corporate settings as calling for a different set of social norms than an abstract laboratory setting, they may not view cheating on a verbal agreement as unethical behavior that merits an angry response. This would obviously affect our results. Finally, almost all business decisions are made in a group context and within an organizational hierarchy. Individuals in these settings may feel more responsible to other people within their corporation than to a person in another firm and hence be less sensitive to being admonished for cheating.

More generally, caution is always needed when generalizing results with a specific population and specific stimuli (payoffs, experimental materials, physical location, etc.). Additional research is needed examining how much our results change as the experimental environment and population change. This is a major component of our ongoing research.

Our paper raises methodological issues concerning the study of communication in games. Studying communication with a limited message space is appealing because of the tight control and ease of analysis it provides, but our results point to problems with this methodology. One concern is that a limited message space may inadvertently exclude critical types of messages. Illustrating this point, we ran a follow-up experiment that modified the $P I C$ treatment to allow for renegotiation: each player is allowed to send a message about their intended action for Period 2 after the outcome for Period 1 has been observed. This has little effect, neither increasing nor decreasing collusion relative to PIC. Even stronger, Andersson and Wengström (2010) find lower cooperation with renegotiation in a similar experiment with limited message spaces. These results obviously differ from our findings in the PChat and RChat treatments. A plausible cause for this difference, especially in our followup study where the population and games are held fixed, is the unavailability of verbal punishments in the limited message space experiments. Comparing the PlC and PChat treatments illustrates a more subtle problem - using a limited message space may limit subjects' ability to effectively use a given message space. Threats of punishment with Low are available in both treatments but only used in PChat. The preceding observations suggest that using a limited message space to study how communication affects cooperation risks missing important features of how communication functions in the more natural context of a conversation.

We view our work as a complement to field work studying the transcripts of communication between colluding firms (e.g., Genesove and Mullin 2001). Field data on communication and collusion comes from firms that were sufficiently successful at colluding to warrant prosecution and sufficiently indiscrete (or possibly unlucky) to get caught, but in the lab we observe the full population, including firms who try to collude and fail. The controlled environment of the lab also allows us to manipulate what types of communication are available to our subjects, for example turning the ability to renegotiate on or off. Players in the field are presumably fairly experienced at the game being played, but in the lab we get to see the learning process as subjects gain experience by playing the game repeatedly. Lab experiments cannot 
match the verisimilitude of field data, but the richness of the data and the ability to study nonnaturally occurring communication structures make them a valuable tool for understanding the relationship between communication and collusion.

On a broad level our results highlight the importance of making a sharp distinction between explicit and tacit collusion in antitrust policy as suggested by Whinston (2006). It is possible to achieve tacit collusion in the lab, but direct communication makes collusion easier. More importantly, our experiments point to the types of communication that should be of particular concern in antitrust enforcement. Calls for collusion in isolation may not be terribly effective. What truly matters is laying out a punishment for failure to stick to a collusive agreement.

\section{APPENDIX}

One of the central conclusions of our paper is that the effect of explicit threats is far greater than any other type of message in the PChat treatment. This relies on the results of the $P$ Chat regression from Table 4 . Table 5 reports regressions testing the robustness this result.

The dataset for these regressions, unless otherwise stated, is all observations from the PChat treatment where the subjects reached an agreement. The dependent variable is a dummy for whether a player cheated on their agreement. We report parameter estimates, not marginal effects. Table 5 only reports the critical estimate, the effect of receiving an explicit threat, but full copies of the regression output are contained in the online appendices.

Model 1 repeats the PChat regression from Table 4. In the text we note that the effect of including controls for sent messages on the estimated effects of received messages is minimal (indicating there is little omitted variable bias). This point is confirmed by Model 2 which removes the controls for sent messages. The parameter estimate for receiving an explicit threat is slightly reduced, but remains easily significant at the 1 percent level, and the marginal effect is slightly reduced to 25 percent in Model 2 as opposed to 26 percent in Model 1. While not shown here, the effect on the other estimates for received messages is also minimal. We believe including the sent messages is the right choice, especially since the resulting estimates are interesting in their own right, but our conclusions vis-à-vis received messages are robust to whether or not controls for sent messages are included.

Another reason the results of Model 1 might not be robust is uncontrolled individual effects. Model 1 includes multiple features designed to control for individual effects: standard errors are corrected for clustering at the individual level and controls are included for the player's behavior in Rounds 1-10 and their opponent's behavior in Rounds 1-10. Going even further, we can include fixed effects in the regression to control for individual effects. Doing this within the framework of a probit is problematic because many subjects either never or always cheat, so we move to a linear probability model to avoid dropping large numbers of observations. Model 3 includes fixed effects for the subject making choices and Model 4 also contains fixed effects for their opponents. In both cases the standard errors are corrected for clustering at the subject level, correcting for the heteroskedasticity associated with use of a linear probability model. Model 3 drops the control for 
Table 5-Alternative Regressions on Effect of Explicit Threats in PCHaT

\begin{tabular}{lccccc}
\hline \hline & Model 1 & Model 2 & Model 3 & Model 4 & Model 5 \\
\hline Subject fixed effects & & & $\checkmark$ & $\checkmark$ & $\checkmark$ \\
Opponent fixed effects & & & $\checkmark$ & $\checkmark$ \\
IV & & & & $\checkmark$ \\
Observations & 626 & 626 & 626 & 626 & 525 \\
Model type & Probit & Probit & $\begin{array}{c}\text { Linear } \\
\text { probability }\end{array}$ & $\begin{array}{c}\text { Linear } \\
\text { probability }\end{array}$ & $\begin{array}{c}\text { Linear } \\
\text { probability }\end{array}$ \\
Received & & & $-0.220^{* * * *}$ & $-0.292^{* * *}$ & $-0.602 *$ \\
$\quad$ explicit threat & $-0.808 * * *$ & $-0.767^{* * *}$ & $(0.064)$ & $(0.084)$ & $(0.360)$ \\
\hline
\end{tabular}

*** Significant at the 1 percent level.

** Significant at the 5 percent level.

* Significant at the 10 percent level.

the player's behavior in Rounds 1-10 since it is collinear with the fixed effect, and Model 4 drops this and the control for the opponent's behavior in Rounds 1-10 for the same reason. For both Model 3 and Model 4, receiving an explicit threat significantly reduces the likelihood of cheating. In both cases the estimated marginal effect of receiving an explicit threat is similar to the 26 percent estimated for Model 1. Adding fixed effects does not affect our primary conclusion that explicit threats are by far the most important type of message in determining whether players will cheat on a collusive agreement.

A final concern for Model 1 is that the received messages are endogenousto be precise, the received messages may be correlated with the error term. The most obvious sources of such correlation are failure to control for individual effects or the effect of sent messages. We hope that at this point it is apparent that these are unlikely to cause the estimated effect of receiving an explicit threat. However, it never hurts to be extra careful. The only channel through which a subject can affect the messages he receives is the messages he sends, since no other interaction between subjects occurs prior to Period 1, but a cautious reader could argue that messages contain nuances that our coding cannot capture so that controlling for commonly coded sent messages is not sufficient. It can also be argued that because sent messages are potentially endogenous, the estimates for received messages will be biased. Given that the results for Models 1 and 2 are quite similar, this is unlikely, but only an instrumental variables approach can adequately address concerns about endogeneity. Model 5 therefore presents an "all of the above" approach to establishing whether there is a causal relationship between cheating and receiving explicit threats. This is a two-stage least squares model. As instruments for the received messages we use the messages sent by the opponent in the previous round, the messages received by the opponent in the previous round, and the opponent's outcome in the previous round. We drop any observations where the same subjects were matched for the previous round, so a subject's opponent's actions and outcomes from the previous round should be uncorrelated with the subject's current error term. All Round 11 observations are dropped since there are no previous round messages to use as instruments. To control for individual effects, fixed effects are included for a 
subject and his opponent. Once again this is a linear probability model. No controls are included for sent messages since these are endogenous.

Model 5 once again yields the result that receiving an explicit threat has a statistically significant effect on reducing cheating. While this effect is only significant at the 10 percent level, explicit threats are the only category of received message with any significant effect. We do not think that the instrumental variables approach taken by Model 5 is the best way to study the data. A large percentage of the data is discarded, a great deal of power is lost through the use of instruments, and the magnitude of the parameter for explicit threats is implausible. Nonetheless, we feel that it is worthwhile showing that the extra step of instrumenting for received messages does not affect our main conclusion: there is a causal relationship between receiving an explicit threat and cheating on collusive agreements.

\section{REFERENCES}

Abreu, Dilip. 1988. "On the Theory of Infinitely Repeated Games with Discounting.” Econometrica 56 (2): 383-96.

Abreu, Dilip, David Pearce, and Ennio Stacchetti. 1990. "Toward a Theory of Discounted Repeated Games with Imperfect Monitoring." Econometrica 58 (5): 1041-63.

-Abreu, Dilip, David Pearce, and Ennio Stacchetti. 1993. "Renegotiation and Symmetry in Repeated Games." Journal of Economic Theory 60 (2): 217-40.

Andersson, Ola, and Erik Wengström. 2010. "More Communication, Less Cooperation: Experimental Evidence from Multi-stage Games." http://project.nek.lu.se/publications/workpap/Papers/ WP07_4.pdf.

Aumann, Robert. 1990. "Nash Equilibria are not Self-Enforcing." In Economic Decision-Making: Games, Econometrics and Optimisation: Contributions in Honour of Jacques H. Drèze, edited by J. J. Gabszewicz, J. F. Richard, and L. A. Wolsey, 201-206. Amsterdam: Elsevier.

-Benoit, Jean-Pierre, and Vijay Krishna. 1985. "Finitely Repeated Games.” Econometrica 53 (4): 905-22.

- Benoît, Jean-Pierre, and Vijay Krishna. 1993. "Renegotiation in Finitely Repeated Games." Econometrica 61 (2): 303-23.

- Bernheim, B. Douglas, Bezalel Peleg, and Michael D. Whinston. 1987. "Coalition-Proof Nash Equilibrium I. Concepts." Journal of Economic Theory 42 (1): 1-12.

- Bernheim, B. Douglas, and Debraj Ray. 1989. "Collective Dynamic Consistency in Repeated Games." Games and Economic Behavior 1 (4): 295-326.

- Blume, Andreas, and Andreas Ortmann. 2007. "The effects of costless pre-play communication: Experimental evidence from games with Pareto-ranked equilibria." Journal of Economic Theory 132 (1): 274-90.

Brandts, Jordi, and David J. Cooper. 2007. "It's What You Say, Not What You Pay: An Experimental Study of Manager-Employee Relationships in Overcoming Coordination Failure." Journal of the European Economic Association 5 (6): 1223-68.

- Brosig, Jeannette, Joachim Weimann, and Axel Ockenfels. 2003. "The effect of communication media on cooperation." German Economic Review 4 (2): 217-41.

Cason, Timothy N., and Vai-Lam Mui. 2009. "Coordinating Resistance through Communication and Repeated Interaction." Unpublished.

-Charness, Gary, and Martin Dufwenberg. 2006. "Promises and Partnership." Econometrica 74 (6): 1579-1601.

-Charness, Gary, and Martin Dufwenberg. 2010. "Bare Promises." Economics Letters 107 (2): 281-83.

- Cohen, Jacob. 1960. "A Coefficient of Agreement for Nominal Scales." Educational and Psychological Measurement 20 (1): 37-46.

Cooper, Russell, Douglas V. DeJong, Robert Forsythe, and Thomas W. Ross. 1992. "Communication in Coordination Games." Quarterly Journal of Economics 107 (2): 739-71.

Cooper, David J., and Kai-Uwe Kühn. 2013. "Communication and Collusion: The Effects of Game Length and Message Space." Unpublished.

- Cooper, David J., and Kai-Uwe Kühn. 2014. "Communication, Renegotiation, and the Scope for Collusion: Dataset." American Economic Journal: Microeconomics. http://dx.doi.org/10.1257/ mic.6.2.247. 
Dal Bó, Pedro. 2005. "Cooperation under the Shadow of the Future: Experimental Evidence from Infinitely Repeated Games." American Economic Review 95 (5): 1591-1604.

-Dal Bó, Pedro, and Guillaume R. Fréchette. 2011. "The Evolution of Cooperation in Infinitely Repeated Games: Experimental Evidence." American Economic Review 101 (1): 411-29.

-Dawes, Robyn M., Jeanne McTavish, and Harriet Shaklee. 1977. "Behavior, Communication, and Assumptions about other People's Behavior in a Commons Dilemma Situation." Journal of Personality and Social Psychology 35 (1): 1-11.

Dawes, Robyn M., Alphons J. C. Van De Kragt, and John M. Orbell. 1988. "Not me or thee but we: The importance of group identity in eliciting cooperation in dilemma situations: Experimental manipulations." Acta Psychologica 68 (1-3): 83-97.

-Duffy, John, and Jack Ochs. 2009. "Cooperative Behavior and the Frequency of Social Interaction." Games and Economic Behavior 66 (2): 785-812.

-Farrell, Joseph, and Eric Maskin. 1989. "Renegotiation in repeated games." Games and Economic Behavior 1 (4): 327-60.

-Farrell, Joseph, and Matthew Rabin. 1996. “Cheap Talk.” Journal of Economic Perspectives 10 (3): $103-18$.

-Fischbacher, Urs. 2007. "z-Tree: Zurich Toolbox for Ready-made Economic Experiments." Experimental Economics 10 (2): 171-78.

Fréchette, Guillaume R., and Sevgi Yuksel. 2013. "Infinitely Repeated Games in the Laboratory: Four Perspectives on Discounting and Random Termination." https://files.nyu.edu/gf35/public/print/ Frechette_2013a.pdf.

-Genesove, David, and Wallace P. Mullin. 2001. "Rules, Communication, and Collusion: Narrative Evidence from the Sugar Institute Case." American Economic Review 91 (3): 379-98.

Harsanyi, John C., and Reinhard Selten. 1988. A General Theory of Equilibrium Selection in Games. Cambridge: MIT Press.

Holt, Charles A., and Douglas Davis. 1990. "The Effects of Non-binding Price Announcements on Posted-Offer Markets." Economics Letters 34 (4): 307-10.

-Isaac, R. Mark, and James M. Walker. 1988. "Group Size Effects in Public Goods Provision: The Voluntary Contributions Mechanism." Quarterly Journal of Economics 103 (1): 179-99.

-Kerr, Norbert L., and Cynthia M. Kaufman-Gilliland. 1994. "Communication, Commitment, and Cooperation in Social Dilemma." Journal of Personality and Social Psychology 66 (3): 513-29.

-Masclet, David, Charles Noussair, Steven Tucker, and Marie-Claire Villeval. 2003. "Monetary and Nonmonetary Punishment in the Voluntary Contributions Mechanism." American Economic Review 93 (1): 366-80.

-Roth, Alvin E., and J. Keith Murnighan. 1978. "Equilibrium behavior and repeated play in prisoner's dilemma." Journal of Mathematical Psychology 17 (2): 189-98.

van Damme, Eric. 1989. "Renegotiation-proof equilibria in repeated prisoners' dilemma." Journal of Economic Theory 47 (1): 206-17.

Van Huyck, John B., Raymond C. Battalio, and Richard O. Beil. 1990. "Tacit Coordination Games, Strategic Uncertainty, and Coordination Failure." American Economic Review 80 (1): 234-48.

Whinston, Michael D. 2006. Lectures on Antitrust Economics. Cambridge: MIT Press

- Xiao, Erte, and Daniel Houser. 2005. "Emotion Expression in Human Punishment Behavior." Proceedings of the National Academy of Sciences 102 (20): 7398-7401. 
This article has been cited by:

1. Pedro Dal Bó, Guillaume R. Fréchette. 2018. On the Determinants of Cooperation in Infinitely Repeated Games: A Survey. Journal of Economic Literature 56:1, 60-114. [Abstract] [View PDF article] [PDF with links]

2. Marco Battaglini, Salvatore Nunnari, Thomas R. Palfrey. 2016. The Dynamic Free Rider Problem: A Laboratory Study. American Economic Journal: Microeconomics 8:4, 268-308. [Abstract] [View PDF article] [PDF with links]

3. Yu Awaya, Vijay Krishna. 2016. On Communication and Collusion. American Economic Review 106:2, 285-315. [Abstract] [View PDF article] [PDF with links] 